\title{
Recycling of Waste Cooking Oils: variation of the chemical composition during water treatment
}

\author{
Alberto Mannu*, ${ }^{a}$ Monica Ferro, ${ }^{\text {a }}$ Greta Colombo Dugoni, ${ }^{a}$ Walter Panzeri, ${ }^{a}$ Giacomo Luigi \\ Petretto, ${ }^{b}$ Paolo Urgeghe ${ }^{b}$ and Andrea Mele*a \\ aDepartment of Chemistry, Materials and Chemical Engineering "G. Natta", Politecnico di Milano, \\ Piazza L. da Vinci 32, 20133 Milano, Italy.

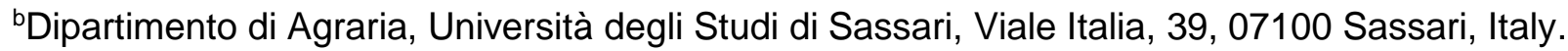

\begin{abstract}
Samples of sunflower Waste Cooking Oils (WCOs) subjected to several cycles of frying were treated with water under four different combinations of temperature and $\mathrm{pH}$. The variation of the chemical composition of the oil samples was monitored through headspace Solid-Phase Microextraction (HSSPME) coupled with gas-chromatographic technique (GC), ${ }^{1} \mathrm{H}$ NMR spectroscopy and $\mathrm{ESI}^{+}$mass spectrometry measurements. The possibility to modify the chemical composition of the recycled vegetable oil by tuning the water treatment parameters was exploited for the designing of a miniplant for the production of bio-lubricants. A full description of the proposed prototype is also reported.
\end{abstract}

Keywords: Waste Cooking Oil, volatiles, ${ }^{1} \mathrm{H}$ NMR, mass spectrometry, prototype. 


\section{Introduction}

During the last decades, the efforts toward the developing of new sustainable green process has grown exponentially. One of the more recent examples of such tendency is represented by the Waste Cooking Oils (WCOs) management ${ }^{1}$, which has been object of new international regulations and became of interest as raw material for several private companies. In Europe and in few other countries, WCOs are collected by the urban waste system ${ }^{2}$ and delivered to an authorized recycling point. This by-product of the food chain, is currently considered an economic and green raw material for several industrial processes. ${ }^{3}$ The possibility to easily transform the used vegetable oil in valuable goods combined with the necessity to avoid its dispersion in the environment ${ }^{4}$ and with its availability in very large amounts, ${ }^{5}$ pushed many local industries to invest in WCOs processing. The most common utilizations of recycled WCO include the bio-lubricants production, ${ }^{6,7,8,9}$ the employment as animal feed, ${ }^{10,11}$ and the energy production (direct burning ${ }^{12,13}$ or as fuel ingredient ${ }^{14,15,16,17}$ ). In addition, some recent application of recycled WCOs involve the production of eco-friendly biodegradable solvents, ${ }^{18}$ the use as additive for bio-asphalts and bio-concrete, ${ }^{19,20}$ and its exploiting as non-aqueous gas sorbents devices. ${ }^{21,22}$ Independently to the specific production, WCOs are usually pre-treated, sometimes by the same collectors, and transformed in regenerated low quality oleic derivative. This first stage of recycling gives a product with a low market values, which is sold to other industries for further transformations.

This first treatment usually includes washing the WCO with water and/or filtering it on cellulose papers or bentonite pads. The first step is also called degumming, ${ }^{23}$ and is borrowed from the industry of edible oils, which are washed in order to remove wax, pigments and metal traces. ${ }^{24,25,26}$ From the other side, the filtration procedure, also known as clarification step, was originally employed in the recycling of mineral oils and permits to decrease of the density and the viscosity and to enhance the color properties of the crude oil by removing selected polar compounds and by separating metal traces and other solids..$^{27,28,29}$ The variation of the chemical composition of WCOs depending on the degumming or filtration has been seldom studied. ${ }^{30}$ It has been found that the volatile composition of crude WCOs is mainly composed by Free Fatty Acids (FFA) and degradation products with respect to the parent edible vegetable oils, and that such composition change depending on the kind of the bentonite employed for the filtering treatment. ${ }^{31,32}$

When WCOs are recycled for non-bio-diesel applications the treatment with water and the filtration represent the main steps in the vegetable oil regeneration process. In particular, the chemicalphysical properties of the recycled oil are sensitive to the conditions of the water. For the best of our knowledge the recycling process of WCO for obtain bio-lubricant or bio-solvents has not been yet described and no report on the influence of the main parameters of the degumming on the chemical composition of the oil has been published so far.

Herein we present for the first time, a detailed description of the variation of the chemical composition of sunflower oils employed for frying ${ }^{33}$ which have been subjected to four different type of water 
treatment. Commercial, crude and refined oils were characterized by headspace Solid-Phase Microextraction (HS-SPME) coupled with gas-chromatographic technique, by ${ }^{1} \mathrm{H}$ NMR and by mass spectrometry (MS-ESI). The main differences between the samples were highlighted though multivariate statistical treatment of the data obtained. In addition, the design of a prototype based on the results of the present study for the production of regenerated vegetable oil is described and commented.

\section{Materials and Methods}

\subsection{Starting materials}

Sunflower oil was considered for the present study and samples of edible oil were purchased by ZUCCHI. Samples of commercial oil M1 were subjected to several frying cycles involving different kind of foods and the corresponding Waste Cooking Oil WCO1 was employed for the water treatments.

\subsection{Degumming procedure}

In a $200 \mathrm{~mL}$ flask, $50 \mathrm{~g}$ of WCO1 and $50 \mathrm{~g}$ of water were mixed together and stirred for 3 hours at the selected temperature. Two sets of $\mathrm{pH}$ values and temperatures were considered: $\mathrm{pH} 3$ and 9 and $30^{\circ} \mathrm{C}$ and $80^{\circ} \mathrm{C}$. The water solutions at $\mathrm{pH} 3$ were prepared by diluting a $33 \% \mathrm{HCl}$ solution with distilled water, while the solutions at $\mathrm{pH} 9$ were obtained by adding $\mathrm{NaOH}$ to deionized water. The $\mathrm{pH}$ was measured with a $\mathrm{pH}$-meter XS Instruments.

\subsection{Headspace Solid-Phase Microextraction (HS-SPME)}

A $100 \mu \mathrm{m}$ PDMS/DVB/CAR (Polydimethylsiloxane/Divinylbenzene/Carboxen) coated fibre 50/30 Stableflex (Supelco, Sigma Aldrich, St. Louis, Mo., USA) was preconditioned prior to use at $270{ }^{\circ} \mathrm{C}$ for $1 \mathrm{~h}$ in a Gerstel MPS bake-out station, according to the manufacturer's instructions. $5 \mathrm{~g}$ of sample were placed in a $20 \mathrm{~mL}$ SPME vial, $75.5 \times 22.5 \mathrm{~mm}$, that was tightly closed with a septum and allowed to equilibrate for $5 \mathrm{~min}$ at $60{ }^{\circ} \mathrm{C}$. The preconditioned fibre was then exposed to the headspace. The extraction time was fixed at $30 \mathrm{~min}$, based on a previous optimization. All experiments were carried out under constant agitation at $250 \mathrm{rpm}$. After the extraction, the fibre was desorbed for $2 \mathrm{~min}$ into a Gerstel CIS6 PTV injector operating at $250^{\circ} \mathrm{C}$ in a splitless injection mode. Results were reported as absolute peak area $\left(\times 1 \mathrm{E}^{6}\right)$

\subsection{GC-MS analysis}

For the GC-MS analysis an Agilent 7890 GC equipped with a Gerstel MPS autosampler, coupled with an Agilent 7000C MSD detector were used. The chromatographic separation was performed on a VF-Wax $60 \mathrm{~m} \times 0.25 \mathrm{~mm}$ i.d., $0.5 \mu \mathrm{m}$ film thickness column (Agilent), employing the following temperature program: $40^{\circ} \mathrm{C}$ hold for $4 \mathrm{~min}$, then increased to $150^{\circ} \mathrm{C}$ at a rate of $5.0^{\circ} \mathrm{C} / \mathrm{min}$, held for 
$3 \mathrm{~min}$ then increased to $240^{\circ} \mathrm{C}$ at a rate of $10^{\circ} \mathrm{C} / \mathrm{min}$, and finally held for $12 \mathrm{~min}$. Helium was used as the carrier gas at a constant flow of $1 \mathrm{~mL} / \mathrm{min}$. The data was analyzed using a MassHunter Workstation B.06.00 SP1, with identification of the individual components (Table 1) performed by comparison with the co-injected pure compounds and by matching the MS fragmentation patterns and retention indices with the built in libraries or literature data or commercial mass spectral libraries (NIST/EPA/NIH 2008; HP1607 purchased from Agilent Technologies).

\subsection{Retention indexes}

A hydrocarbon mixture of n-alkanes (C9-C22) was analyzed separately under the same chromatographic conditions described in GC-MS section to calculate the retention indexes with the generalized equation by Van del Dool and Kartz, ${ }^{34} I_{x}=100\left[\left(t_{x}-t_{n}\right) /\left(t_{n+1}-t_{n}\right)+n\right]$. Where $t$ is the retention time, $x$ is the analyte, $n$ is the number of carbons of alkane that elutes before analyte and $n+1$ is the number of carbons of alkane that elutes after the analyte.

\subsection{NMR analysis}

${ }^{1} \mathrm{H}$ NMR spectra were recorded on a Bruker AV500 spectrometer operating at $500 \mathrm{MHz}$ for the proton nucleus. All the experiments were performed at $305 \mathrm{~K}$ with the following acquisition parameters: time domain $32 \mathrm{~K}$, relaxation delay $3 \mathrm{~s}, 128$ scans and a spectral width of $12 \mathrm{ppm}$. All the samples were analyzed in pure form, using a coaxial tube with deuterated DMSO for lock.

\subsection{Mass spectrometry}

The ESI-MS experiments were performed using a Bruker Esquire 3000 PLUS (ESI Ion Trap LC/MSn System), equipped with an ESI source and a quadrupole ion trap detector (QIT). Acquisition parameters: needle: $4.5 \mathrm{kV}, \mathrm{N}_{2}$ flow rate: $10 \mathrm{~L} \mathrm{~h}^{-1}$, cone voltage: $40 \mathrm{~V}$, Scan resolution and range: $13000(\mathrm{~m} / \mathrm{z}) \mathrm{s}^{-1}$ over the mass range $\mathrm{m} / \mathrm{z} 35-500$, by direct infusion of methanol solution of compounds at rate: 4 microliter $\mathrm{min}^{-1}$.

\subsection{Statistical analysis}

The Principal Component Analysis (PCA) of the data relative to the chemical composition of the samples obtained by MS-ESI-IT spectrometry was conducted. Each sample was analyzed in triplicate to define a matrix $\mathrm{m} \times \mathrm{n}$ where $\mathrm{m}$ were the samples and $\mathrm{n}$ were the variables. The data were centered and autoscaled before the PCA. All PCA analyzes were performed with Metaboanalyst 4.0. ${ }^{35}$

\section{Result and Discussion}

In a previous paper, we studied through Design Of Experiments (DOE) and multivariate analysis the impact of acidic degumming of WCOs samples on their color, density and flash point, describing the 
influence of the temperature, stirring time, oil/water ratio, and $\mathrm{pH}^{30} \mathrm{We}$ observed that the most influencing parameters during water treatment of WCOs are temperature and $\mathrm{pH}$, while the oil/water ratio and the stirring time influence less the physical characteristics of the recycled oil. Taking into consideration our previous results, herein we investigate and report on the effect of $\mathrm{pH}$ and temperature on the chemical composition of sunflower waste oil. A temperature range from $30^{\circ} \mathrm{C}$ to $80^{\circ} \mathrm{C}$ and a pH window between 3 and 9 were considered. Samples of Waste Cooking Oil were treated with water at a specific $\mathrm{pH}$ and temperature ${ }^{36}$, and the corresponding chemical compositions determined by GC/MS analysis, ${ }^{1} \mathrm{H}$ NMR spectroscopy and $\mathrm{ESI}^{+}$mass spectrometry were compared. In order to test the level of regeneration of the proposed procedure, the same analyses were performed on samples of the original matrix, the commercial sunflower oil M1 (Table 1).

Table 1: nomenclature of the samples considered in the present study.

\begin{tabular}{cl}
\hline Sample & Condition $^{\mathrm{a}}$ \\
\hline M1 & $\begin{array}{l}\text { Sunflower commercial oil } \\
\text { M1 subjected to several frying cycles }\end{array}$ \\
& \\
01 & WCO1 after treatment at $\mathrm{pH}=3$ and $\mathrm{T}=25^{\circ} \mathrm{C}$ \\
$\mathbf{0 2}$ & WCO1 after treatment at $\mathrm{pH}=9$ and $\mathrm{T}=25^{\circ} \mathrm{C}$ \\
$\mathbf{0 3}$ & WCO1 after treatment at $\mathrm{pH}=3$ and $\mathrm{T}=80^{\circ} \mathrm{C}$ \\
& WCO1 after treatment at $\mathrm{pH}=9$ and $\mathrm{T}=80^{\circ} \mathrm{C}$ \\
\hline
\end{tabular}

aRatio oil/water 70/30 in volume, time of stirring 3 hours.

\subsection{Volatile Organic Compounds (VOCs)}

All the samples indicated in Table 1 were analyzed by headspace Solid-Phase Microextraction (HSSPME) coupled with gas-chromatographic technique in order to characterize the volatile fraction. Thirty components were identified as reported in Table $2 .{ }^{37}$

Table 2: main components detected by Gas-Chromatography in samples of edible oil M1, fried oil WCO1, and treated samples $01,02,03$, and 04 . The values expressed are the result of the average value of three independent replicates \pm SD of absolute TIC peak area (1E6).

\begin{tabular}{ccccccccc}
\hline Entry & Retention time & M1 & WCO1 & O1 & O2 & O3 & O4 & Analyte \\
\hline 1 & 15,489 & n.d. & 2,7287 & 1,9981 & 1,7933 & 0,8577 & 1.8302 & Hexanal \\
2 & 19,009 & n.d. & 0,7503 & 0,2685 & 0,3263 & - & 0,6493 & Heptanal \\
3 & 19,573 & n.d. & 0,7684 & - & 0,4901 & - & 0,4494 & Limonene \\
4 & 20,354 & n.d. & 1,8623 & 1,0677 & 1,2202 & - & 0,4494 & Furan, 2-pentyl- \\
5 & 25,183 & n.d. & 4,0382 & 3.8689 & 4.0172 & 2.8489 & 2.4767 & Nonanal \\
6 & 26,086 & n.d. & 0,8284 & 0,6770 & 0,8705 & 0,4485 & 0,7353 & 1-octen-3-ol \\
7 & 27,052 & n.d. & 0,6783 & - & 0,5719 & 0,0791 & 0,1544 & Furfural \\
8 & 29,158 & n.d. & 0,5625 & 0,4577 & 0,4587 & 0,1700 & 0,5569 & Cyclohexanol,-dimethyl- (not identified isomer) \\
9 & 29,475 & n.d. & 0,4908 & 0,4230 & 0,4550 & 0,2407 & 0,2646 & benzaldheyde
\end{tabular}




$\begin{array}{cccccccc}29,692 & \text { n.d. } & 0,6348 & 0,6042 & 0,5006 & 0,4925 & 0,7101 & \text { 2-Nonenal } \\ 32,182 & \text { n.d. } & - & - & - & - & - & \text { 2-Furan-methanol } \\ 32,423 & \text { n.d. } & 1,4988 & 1,4236 & 1,4708 & 1,3255 & 1,5066 & \text { 2-Decenal } \\ 34,587 & \text { n.d. } & 1,0504 & 1,0649 & 1,0810 & 1,0060 & 0.6942 & \text { 2-Undecenal } \\ 34,79 & \text { n.d. } & 1,2092 & 1,1551 & 1,1331 & 1,0718 & 1,0759 & \text { 2,4-Decadienal, (not identified isomer) } \\ 35,929 & \text { n.d. } & - & - & - & - & 0,4468 & \text { 2,4-Decadienal, (not identified isomer) }\end{array}$

In the volatile fraction of edible oil $\mathbf{M} \mathbf{1}$ cannot be detected any relevant analyte. This is in agreement with the fact that edible oils are usually subjected to a deodorization process, which remove most of the volatile compounds. ${ }^{38,39}$

Samples of fried WCO1 resulted enriched in many classes of compounds, such as aldehydes, alcohols, dienes. These compounds result from the frying process and in particular from the oxidation of triacylglycerols and via the Maillard process (nitrogen-containing compounds). Some terpenes could also be found, probably arising from food leaching. ${ }^{40,41}$ The presence of alkanes is a consequence of the pyrolysis process of the free fatty acids occurring during deep frying. ${ }^{42}$ Also, some products resulting from the autoxidation of the linolenic acid were detected as dienals (e.g. 2,4-decadienal), and alkenals such as 2-undecenal, 2-decenal, 2-noenal, were also detected. ${ }^{43}$

The data reported in table 2 highlight two main trends. First, the treatment at room temperature, independently of the $\mathrm{pH}$, is less effective with respect to the same procedure at $80^{\circ} \mathrm{C}(\mathbf{0 1}$ vs $\mathbf{0 3}$, and 02 vs 04$)$. Second, the effect of $\mathrm{pH}$ is evident: the acidic treatment results to be more effective in reducing the amount of all the volatile compounds detected in comparison with the basic treatment. If we compare the two processes at $80^{\circ} \mathrm{C}$ (O3 vs $\left.\mathbf{0 4}\right)$, many compounds reduced with the basic washing are completed removed at $\mathrm{pH} 3$ (heptanal, limonene, furan-2-pentyl, 2,4-decadienal). Finally, all the four processes removed efficiently 2-furan-methanol, detected in the WCO1 samples only.

\subsection{NMR Spectroscopy}

${ }^{1} \mathrm{H}$ NMR spectroscopy has been already exploited for the characterization of frying oils. ${ }^{44,45,46}$ The technique represents a valid alternative to the commonly employed chromatographic methods as it allows the quick analysis of easily prepared samples and the identification of many compounds at the same time. ${ }^{47}$ The application of ${ }^{1} \mathrm{H}$ NMR spectroscopy to the characterization of vegetable oil include: the assessment of the geographical origin of the olive oil, ${ }^{48,49}$ the determination of the fatty acids profile, ${ }^{50,51}$ and the estimation of the quality of waste cooking oils. ${ }^{52}$

${ }^{1} \mathrm{H}$ NMR experiments were performed in order to confirm the differences in the composition of sunflower commercial oil M1, the derived waste WCO1, and the four treated samples 01, 02, 03 and $\mathbf{0 4}$. As expected, the main composition of all the samples analyzed was a mixture of fatty acids (linolenic, linoleic and saturated fatty acids) and unsaturated acids (Figure 1 and Table 1). 


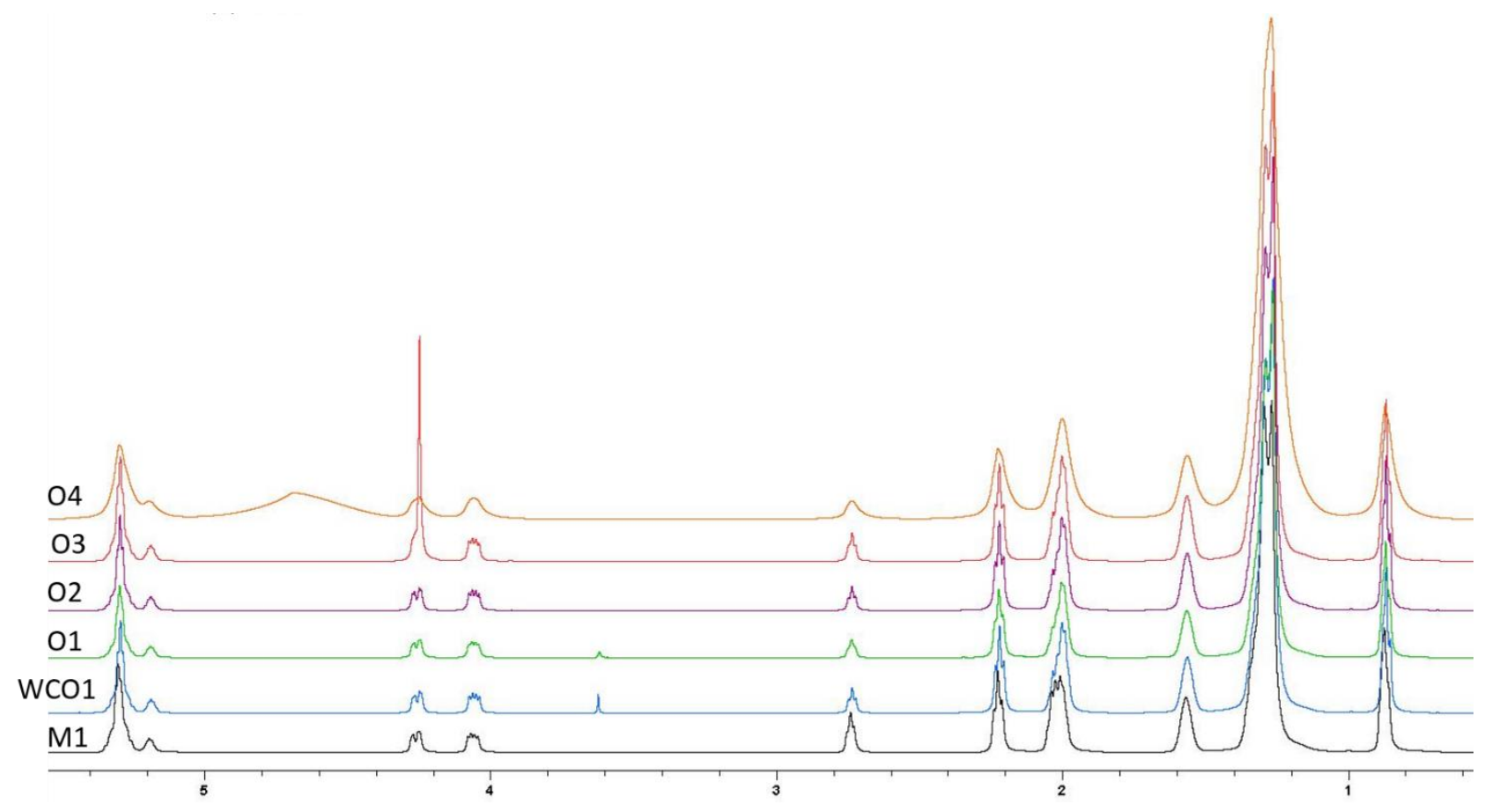

Figure 1: ${ }^{1} \mathrm{H}$ NMR spectra of oil samples edible oil M1, fried oil WCO1, and treated samples 01, 02, 03, and 04.

${ }^{1} \mathrm{H}$ NMR spectra of samples M1, WCO1, 01, O2, 03 and 04 are reported in Figure 1. The characteristic signals of fatty acids and unsaturated acids are present: $-\mathrm{CH}_{3}$ signals at $0.9 \mathrm{ppm},-\mathrm{CH}_{2}$ group of the acyl chains at $1.3 \mathrm{ppm}, 1.6 \mathrm{ppm}$ and $2.3 \mathrm{ppm}$, and the methylenic signals of the unsaturated fatty acids, linolenyl acid and glycerol at 2.0 ppm, 2.2 ppm and between 4.0 and 4.4 ppm, respectively. The triacylglicerols and unsaturated fatty acids can be detected at $5.2 \mathrm{ppm}$ and 5.3 ppm.

According to the methodology proposed by Popescu and coworkers, ${ }^{53}$ it was possible to determine the main composition of the samples analyzed as reported in Table 3.

Table 3: main composition of samples edible oil M1, fried oil WCO1, and treated samples 01, 02, 03, and $\mathrm{O} 4$ observed by ${ }^{1} \mathrm{H}$ NMR spectroscopy. The relative normalized percentage are reported.

\begin{tabular}{ccccccc}
\hline Entry & & Linolenic acid & Linoleic acid & Oleic acid & SFA & IV \\
\hline $\mathbf{1}$ & M1 & $1.9 \%$ & $35.7 \%$ & $46.4 \%$ & $14.0 \%$ & 106 \\
$\mathbf{2}$ & WCO1 & $1.7 \%$ & $19.1 \%$ & $62.2 \%$ & $15.4 \%$ & 91 \\
$\mathbf{3}$ & $\mathbf{0 1}$ & $1.8 \%$ & $17.0 \%$ & $63.7 \%$ & $15.7 \%$ & 88 \\
$\mathbf{4}$ & $\mathbf{0 2}$ & $1.7 \%$ & $17.6 \%$ & $63.6 \%$ & $15.4 \%$ & 89 \\
$\mathbf{5}$ & $\mathbf{0 3}$ & $1.9 \%$ & $18.4 \%$ & $62.1 \%$ & $15.8 \%$ & 90 \\
$\mathbf{6}$ & $\mathbf{0 4}$ & $3.7 \%$ & $14.7 \%$ & $63.4 \%$ & $14.1 \%$ & 85 \\
\hline
\end{tabular}


The relative concentration of the main acids presents in sunflower vegetal oil showed considerable changes after frying: the concentration of linoleic acid drops from $35.7 \%$ of the M1 samples to $19.1 \%$ of WC01. On the other hand, the relative concentration of oleic acid increases from $46.4 \%$ of the edible oil to more than $60 \%$ in WCO1 and in all the treated samples. Treatment of WCO1 at $80{ }^{\circ} \mathrm{C}$ and $\mathrm{pH} 9$ (04) results in an increasing of the relative amount of linolenic acid (entry 6 versus 2-5) and a reducing of the linoleic acid (14.7\% versus $17-19 \%$ of the others). The change in the composition of the oil and in particular of the ration between linoleic and oleic acids as consequence of the frying are reflected in a change of the iodine number, related to the global insaturation (IV). Some undesired products arising from the frying can be detected by NMR. From the expansion of the ${ }^{1} \mathrm{H}$ NMR spectra between 10.5 and 11.8 ppm reported in figure 2 is possible to observe the presence of alkenals, which result from the oxidation of the fatty acids promoted by heat and oxygen (Figure 2, M1 versus WCO1). Errore. II segnalibro non è definito. All the four treatments reduce the amount of detectable aldehydes, even if they are not effective in completely removing them from the crude substrate (Figure 2, spectra 01, 02, 03, and 04).

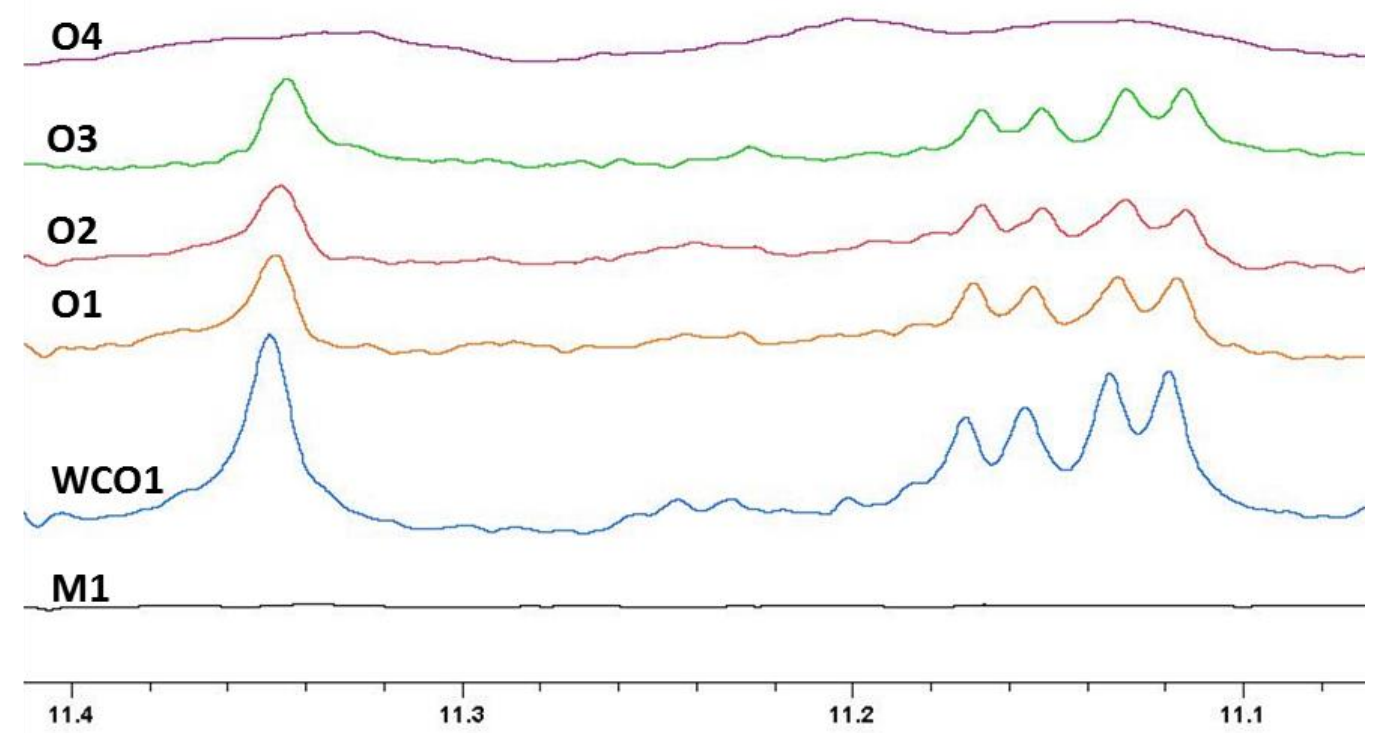

Figure 2: Zoom of ${ }^{1} \mathrm{H}$ NMR spectra of edible oil M1, fried oil WCO1, and treated samples 01, 02, 03, and 04 . Spectral region between 10.5 and 11.8 ppm.

\subsection{Mass spectrometry analysis (MS-ESI)}

Samples of edible oil M1, fried oil WCO1, and treated oils 01, 02, 03 and 04 were analyzed by MS$\mathrm{ESI}^{+}$measurements. The MS-ESI spectra of the different samples are very different and complex as they have a huge amount of signals in the observed region. This makes the visual inspection and comparison of the samples difficult. For this reason, multivariate analysis was used in order to discriminate the samples on the bases on their chemical fingerprint. 
From the GC-MS and ${ }^{1} \mathrm{H}$ NMR data we observed that the main differences in composition between untreated, fried and treated samples were related to the presence of degradation products which are low molecular weight molecules (Figures 3 and 4). Taking this into account and with the aim to verify which treatment is more suitable for the regeneration of the fried oil in terms of removing the decomposition products, the spectral region between $\mathrm{m} / \mathrm{z} 100$ and 500 was subjected to Principal Component Analysis (PCA) (Figures 3 and 4).

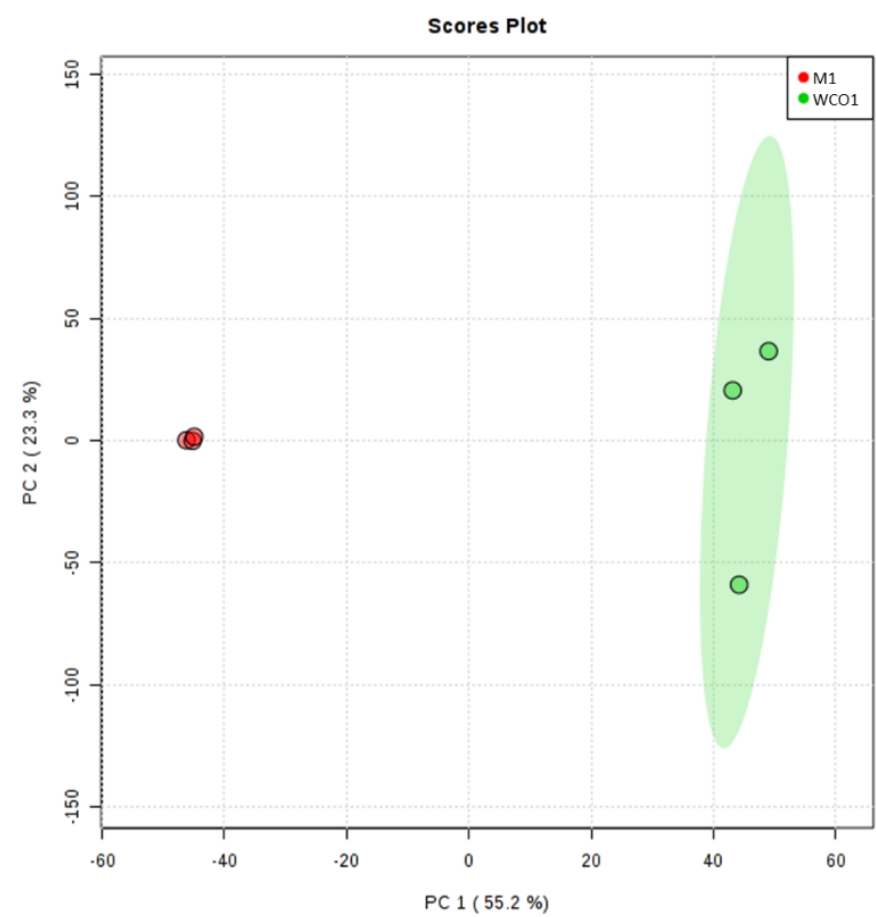

Figure 3: PCA score plot for samples of edible oil M1 (red) and fried oil WCO1 (green).

The MS-ESI normalized data were analyzed using MetaboAnalyst 4.0, an online tool for Multivariate statistical Analysis. The PCA score plot reported in figure 3 shows differences in the chemical composition between the edible oil M1 and the fried one WCO1. The plot reported in Figure 3 confirms that MS/ESI combined with statistical analysis can be used to underline differences and similarity in chemical composition of edible and fried oils. The two principal components PC1 and PC2 explain the $78,5 \%$ of the total variance, respectively for the $55,2 \%$ and $23,3 \%$. The data relative to the edible oil M1 form a small cluster while the fried oil WCO1 points are more separated, thus indicating a greater inhomogeneity of the sample.

In order to measure the degree of purification of every process studied, the data relative to all the samples (including the treated oils 01, 02, 03 and 04) were introduced in the statistical system. The 2D and 3D PCA score plots for the comparison of edible oil M1, fried oil WCO1 and the treated samples 01, $\mathbf{0 2 ,} \mathbf{O} 3$ and $\mathbf{0 4}$ are reported in figure 4. 

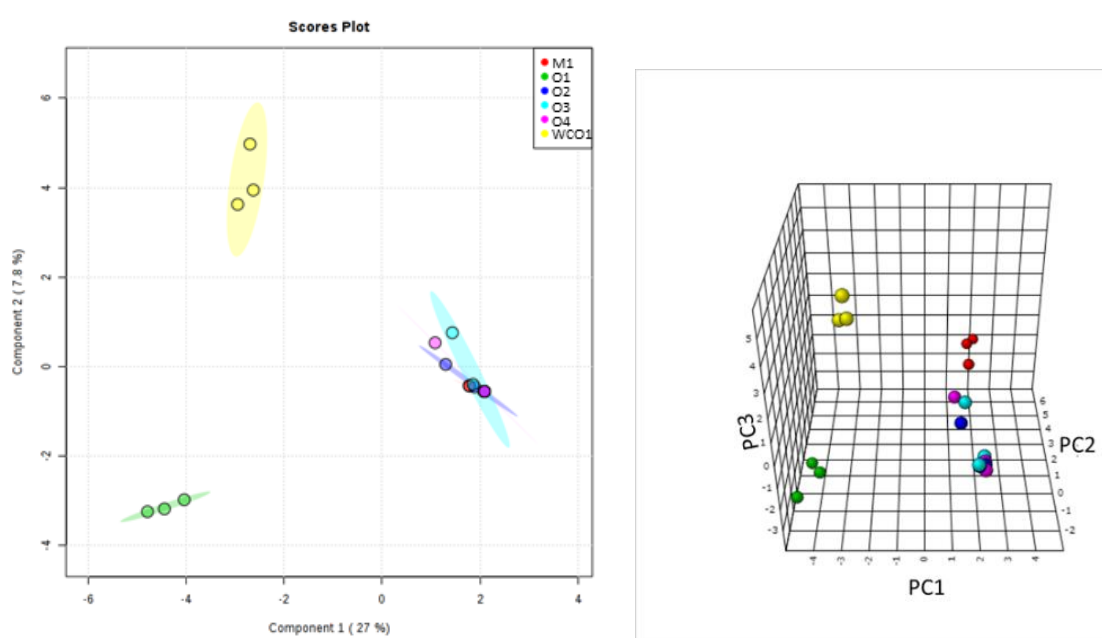

Figure 4: 2D (left) and 3D (right) PCA score plot for comparison of samples M1 (red), WCO1 (yellow), 01 (green), 02 (dark blue), 03 (light blue), and 04 (pink).

Samples 01 form a cluster far from M1 samples and from 02, 03 and 04, clearly indicating that the treatment at $\mathrm{pH} 3$ and room temperature is not as effective as the others in regenerating the frying oil. Conversely, samples $\mathbf{0 2}, \mathbf{O} 3$ and $\mathbf{0 4}$ form a cluster partially overlapped to M1 samples confirming a similarity of these samples and revealing the partial regeneration of the oil. This suggests that $\mathrm{pH} 3$ and $80^{\circ} \mathrm{C}$ and $\mathrm{pH} 9$, at both $25^{\circ} \mathrm{C}$ and $80^{\circ} \mathrm{C}$ are the ideal conditions to clean the WC01 by restoring the original chemical composition of M1. These data are particularly useful if combined with some practical observations. In fact, alkaline treatment of WCO1 leads to the formation of an emulsion due to the saponification of the fatty acids present in the vegetable oil, making any further separation procedures complex. On the contrary, the treatment at $\mathrm{pH} 3$ does not show any evident side-reaction (as saponification), thus allowing an easier separation of the treated oil from the wastewater than the alkaline processes. A careful analysis of the plots reported in figure 4 points out some subtle differences among samples 02,03 and 04 . Oils arising from alkaline treatments (O2 and $\mathbf{0 4}$ ) are almost superimposed in both the components which contribute to the variance (figure 4 left, $2 \mathrm{D}$ plot), while the oil resulting from the acidic process at $80{ }^{\circ} \mathrm{C}(\mathbf{O})$ shows more variability in the component 2 with respect to the other $\mathbf{O 2}$ and $\mathbf{0 4}$. In general, alkaline treated oils are more similar than $\mathbf{0} \mathbf{3}$ to the original edible oil $\mathbf{M} \mathbf{1}$, but the differences are indeed small leading to the conclusion that, considering the collateral saponification process occurring in alkaline media, the ideal conditions for waste cooking oils regeneration are $\mathrm{pH}=3$ and $80^{\circ} \mathrm{C}$.

\subsection{Prototype}

From the data collected in the present study and during our previous investigations, ${ }^{27}$ a proper recycling of WCO should allow to obtain specific characteristics in the recycled material by tuning some process parameters. In particular, the water treatment led to different outcome depending mainly on the temperature and on the $\mathrm{pH}$, while the filtration step improves the color of the 
regenerated oil and helps removing residual aldehydes. Errore. II segnalibro non è definito. Taking in consideration these findings, we designed a modular mini-plant for the recycling of WCOs.

The overall process has been partitioned in three main steps, each one related to a specific working module: the storing and washing module, the filtering module, and the product storing module (Figure 5).

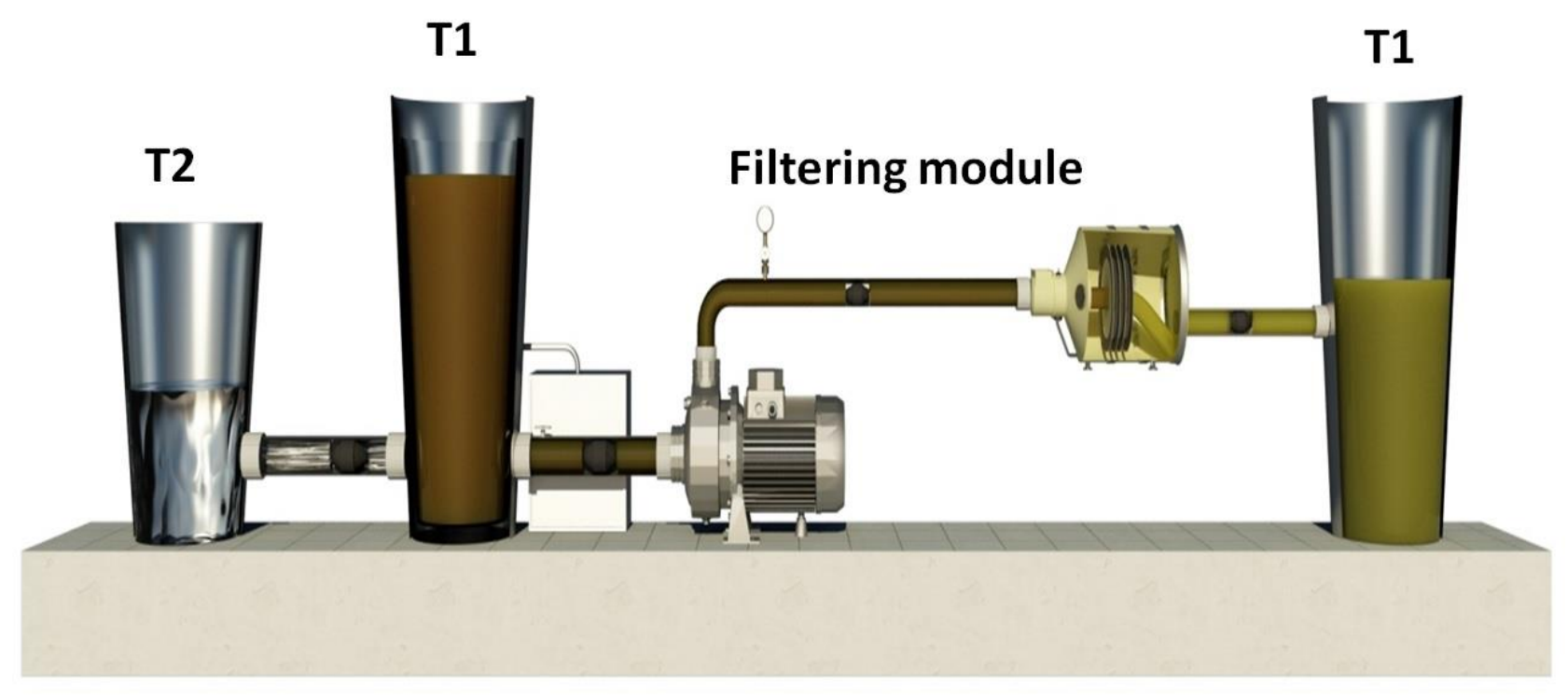

Figure 5: Overview of the mini-plant designed: washing tank (T1), wastewaters decantation tank (T2), filtering module, and storing tank T3.

\subsubsection{Storing and washing module}

The WCO is introduced inside the tank T1 (AISI 304) with a capacity of $50 \mathrm{~L}$ of oil. A first gross filtration $(\geq 100 \mu \mathrm{m})$ take place at this stage thanks to an electronic controlled self-cleaning filter connected to the inlet nozzle. A bag filtering system steel AISI 316 with an integrated electric pump is used. The maximum flow of $100 \mathrm{~L} / \mathrm{min}$. obtaining a laminar motion-based flux (Normal flow filtration), and allowing the oil to pass through the filter perpendicularly to the surface of the septum. The process is monitored by an electronic control every 10 filtering cycles. Once the oil has been introduced in $\mathbf{T 1}$, the suitable amount of water is added.

A system of level probes would be necessary in order to establish the water presence on the bottom of the tank. Two solenoid valves are installed: one for the water discharge and a second one on the opposite side for the washed oil transport to the next module. An additional tap is present for occasional sampling or for emptying the system (e.g. for maintenance).

The temperature of the tank is controlled by a hydraulic system constituted by a serpentine filled with a process fluid passing inside a suitable interspace.

\subsubsection{Separation module}


Once concluded the water treatment, the oil is separated by the water through gravity decantation in the separation module.

The separation module includes a second tank (T2) for the decantation of the wastewater and the connecting pipe. The pipe is made by $\mathrm{DN}=15 \mathrm{~mm}$ steel, while the connections between the two tanks $\mathbf{T} 1$ and $\mathbf{T} 2$ are governed by automatic release systems. This is mandatory for the easy replacing of the tanks. The tank T2 is of AISI 304 type with a total capacity of $30 \mathrm{~L}$. Also, as in the case of T1, a tap is installed for sampling or for the controlled emptying.

\subsubsection{Filtering module}

Washed oil is then filtered with clays in order to remove pigments, metal traces improving its color and the density characteristics.

The filtering module includes one hydraulic pump, a filter filled with porous materials, Errore. Il segnalibro non è definito. and all the corresponding connections (DN $15 \mathrm{~mm}$ ). The pump pushes the oil from the T1 to the filter unit with a maximum pressure of 4 bar. An automatic control which include a shutdown system in the case of excessive pressure will be also installed.

The filtering unit is made by a fiberglass container with a maximum capacity of 3 liters of solid material, connected to the rest of the system by flanged joints. The type of filtration proposed is the "Deep bed filtration", where the oil pass through all the length of the filter. Once exhausted the filtering unit can be easily substituted. The difference between the upstream and the downstream pressure is monitored in order to detect any load loss. As the load losses are related to the grain size of the filtering material, the system also allow to tune the flow pressure depending on the kind of clay employed. The optimization of the filtering procedure has been made considering the equation $\boldsymbol{\Phi}=\Delta \mathbf{P} / \mathbf{R}$ which describes the flux of a fluid that pass through a porous bed. ${ }^{54}$

\subsubsection{Storing module}

The storing area is constituted by the connecting steel pipe (DN $15 \mathrm{~mm}$ ) between the filtering unit and the storing tank T3. The connections between the pipe and the tanks are controlled by a gate sludge system, in order to make easier the disassembly of the module. The T3 steel AISI 304 tank have a maximum capacity of 30 liters and are used for the storing of the regenerated vegetable oils. As for $\mathbf{T} 1$ and $\mathbf{T} 2$, a pipe allow sampling and emptying.

The overall mini-plant is assembled on a wheeled structure with the possibility to remove every single module at the need. The frame of the support structure is constituted by tubular steel cables DN 30 $\mathrm{mm}$ on 4 full rubber wheels. The upper part of the frame has a multilayer base of the type "Okumè" $40 \mathrm{~mm}$, with a flexural strength of $48 \mathrm{MPa}$ (longitudinal) and $16 \mathrm{MPa}$ (transversal). ${ }^{55}$

The modular prototype described is able to treat about 35 liters of WCOs every three hours. In fact, the possibility to change the tank $\mathbf{T 1}$ once the water treatment is concluded allows to treat new oil while the previous washed is stored for decantation. The condition defined through the tests reported above $\left(\mathrm{pH} 3\right.$ and $80^{\circ} \mathrm{C}$ ) reduce the decantation time from days (in the case of alkaline processes, where saponification occurs) to 4-5 hours. In addition, as these conditions avoid the saponification, 
there is no need of adding undesired salts of other additives the emulsion brake down.. The possibility to easily tune the temperature, the stirring time, the oil/water ratio and the water $\mathrm{pH}$, also permits to change the treatment depending on the specific target without any time loss. For instance, waste oils destined to biodiesel can be washed at $\mathrm{pH}=3$ at $25^{\circ} \mathrm{C}$, while waste oils for biolubricants should be regenerated at $\mathrm{pH}=3$ and $80^{\circ} \mathrm{C}$.

\section{Conclusions}

The regeneration of waste cooking oils (WCOs) through water treatment has been discussed and described. The analysis of the variation of the chemical composition of samples of WCOs degummed under different conditions by headspace Solid-Phase Microextraction (HS-SPME) coupled with gaschromatographic technique, ${ }^{1} \mathrm{H}$ NMR and ESI-MS revealed the possibility to tune the characteristics of the recycled oil by changing the temperature and the $\mathrm{pH}$ of the process. From the analysis of the data obtained was evident that working at $\mathrm{pH} 3$ and $80{ }^{\circ} \mathrm{C}$ ensures a good regeneration level of WCO samples avoiding the formation of emulsions which make difficult the subsequent water removal. Also, the possibility to employ the fast ESI-MS technique coupled with PCA analysis for determining a fingerprint of vegetable oils has been revealed, opening to a new industrial application of such procedure (e.g. in the production of biolubricants). A prototype of a mini plant for WCOs recycling, based on the finding described, has been also presented and described. The process optimized allows to fast regenerating waste cooking oils for different applications. 


\title{
5. References
}

\begin{abstract}
${ }_{1}^{1}$ Rodrigues Pereira Ramos, T., Gomes, M.I., Barbosa-Póvoa, A.P., 2013. Planning waste cooking oil collection systems. Waste Management, 33, 1691-1703. https://doi.org/10.1016/..wasman.2013.04.005.

2 http://ec.europa.eu/environment/waste/framework/list.htm.

${ }^{3}$ Borrello, M., Caracciolo, F., Lombardi, A., Pascucci, S., Cembalo, L. 2017. Consumers' Perspective on Circular Economy Strategy for Reducing Food Waste. Sustainability, 9(141), 1-18. https://doi.org/10.3390/su9010141.
\end{abstract}

${ }^{4}$ Li, Y., Jina, Y., Lia, J., Chena, Y., Gongc, Y., Zhang, Y.L.J. 2016. Current Situation and Development of Kitchen Waste Treatment in China. Procedia Environmental Sciences, 31, 40-49. https://doi.org/10.1016/j.proenv.2016.02.006.

${ }^{5}$ http://www.eubia.org/cms/wiki-biomass/biomass-resources/challenges-related-to-biomass/used-cooking-oilrecycling/

${ }^{6}$ Petran, J.; Pedisic, L.; Orlovic, M.; Podolski, S.; Bradac, V. 2008. Biolubricants from natural waste oil and fats. Goriva i maziva, 47, 463-478.

7 Shashidhara, Y.M., Jayaram, S.R. 2010. Vegetable oils as a potential cutting fluid-An evolution. Tribology Intern., 43, 1073-1081. https://doi.org/10.1016/j.triboint.2009.12.065.

${ }^{8}$ Zhang, Y., Dube, M.A., McLean, D.D., Kates, M. 2003. Biodiesel production from waste cooking oil: Process design and technological assessment. Bioresource Technology 89, 1-16. http://dx.doi.org/10.1016/S09608524(03)00040-3.

${ }^{9}$ Hamze, H.; Akia, M.; Yazdani, F. 2015. Optimization of biodiesel production from the waste cooking oil using response surfacemethodology. Process Safety and Environmental Protection, 94, 1-10. https://doi.org/10.1016/j.psep.2014.12.005

10 Salemdeeb, R., zu Ermgassen, E.K.H.J., Kim, M.H., Balmford, A., Al-Tabbaa, A. 2017. Environmental and health impacts of using food waste as animal feed: a comparative analysis of food waste management options. Journal of Cleaner Production, 140, 871-880. https://doi.org/10.1016/j.jclepro.2016.05.049.

11 Tres, A., Bou, R., Guardiola, F., Nuchi, C.D., Magrinya, N., Codony, R. 2013. Use of recovered frying oils in chicken and rabbit feeds: effect on the fatty acid and tocol composition and on the oxidation levels of meat, liver and plasma. Animal, 7(3), 505-517. https://doi.org/10.1017/S1751731112001607

12 Singhabhandhu, A., Tezuka, T. 2010. The waste-to-energy framework for integrated multi-waste utilization: Waste cooking oil, waste lubricating oil, and waste plastics. Energy, 35, 2544-2551. https://doi.org/10.1016/j.energy.2010.03.001

${ }^{13}$ Singhabhandhu, A.; Tezuka, T. 2010. Prospective framework for collection and exploitation of waste cooking oil as feedstock for energy conversion. Energy, 35, 1839-1847. https://doi.org/10.1016/j.energy.2010.01.004 14 Namoco, C.S. Jr., Comaling, V.C., Buna, C.C. Jr. 2017. Utilization of used cooking oil as an alternative cooking fuel resource. ARPN Journal of Engineering and Applied Sciences, 12(2), 435-442. ISSN 1819-6608 ${ }^{15}$ Capuano, D., Costa, M., Di Fraia, S., Massarotti, N., Vanoli, L. 2017. Direct use of waste vegetable oil in internal combustion engines. Renewable and Sustainable Energy Reviews, 69, 759-770. https://doi.org/10.1016/j.rser.2016.11.016

${ }^{16}$ No, S.Y. 2011. Inedible vegetable oils and their derivatives for alternative diesel fuels in Cl engines: A review. Renewable and Sustainable Energy Reviews, 2011, 15, 131-149. 
17 Talebian-Kiakalaieh, A., Amin, N.A.S., Mazaheri, H. 2013. A review on novel processes of biodiesel production from waste cooking oil. Applied Energy, 104, 683-710. https://doi.org/10.1016/j.apenergy.2012.11.061.

18 Panadare, D.C., Rathod, V.K. 2015. Applications of Waste Cooking Oil Other Than Biodiesel: A Review. Iranian Journal of Chemical Engineering, 12(3), 55-76.

19 Sun, D., Lu, T., Xiao, F., Zhu, X., Sun, G. 2017. Formulation and aging resistance of modified bio-asphalt containing high percentage of waste cooking oil residues. Journal of Cleaner Production, 161, 1203-1214. https://doi.org/10.1016/j.jclepro.2017.06.155.

${ }^{20}$ Asli, H., Ahmadinia, E., Zargar, M., Karim, M.R. 2012. Investigation on physical properties of waste cooking oil - Rejuvenated bitumen binder. Construction and Building Materials, 37, 398-405. https://doi.org/10.1016/j.conbuildmat.2012.07.042.

${ }^{21}$ Worthington, M.J.H., Kucera, R.L., Albuquerque, I.S., Gibson, C.T., Sibley, A., Slattery, A.D., Campbell, J.A., Alboaiji, S.F.K., Muller, K.A., Young, J., Adamson, N., Gascooke, J.R., Jampaiah, D., Sabri, Y.M., Bhargava, S.K., Ippolito, S.J., Lewis, D.A., Quinton, J.S., Ellis, A.V., Johs, A., Bernardes, G.J.L., Chalker, J.M. 2017. Laying Waste to Mercury: Inexpensive Sorbents Made from Sulfur and Recycled Cooking Oils. Chem. Eur. J., 23, 16219-16230. https://doi.org/10.1002/chem.201702871.

22 Lhuissier, M., Couvert, A., Amrane, A., Kane, A., Audi, J.-L. 2018. Characterization and selection of waste oils for the absorption and biodegradation of VOC of different hydrophobicities. Chemical Engineering Research and Design, 138, 482-489. https://doi.org/10.1016/j.cherd.2018.08.028.

23 The term "degumming" is usually referred to the removing of gums (mainly phospholipids) in crude vegetable oil. This term can be extended also to used vegetable oils even if they should not contain any phospholipid.

24 Dijkstra, A. J., Van Opstal, M. 1989. The total degumming process. J. Am. Oil Chem. Soc., 66, $1002-1009$. 25 Zufarov, O.; Schmidt, Š.; Sekretár, S. 2008. Degumming of rapeseed and sunflower oils. Acta Chimica Slovaca, 1(1), 321-328.

${ }^{26}$ Gupta, M. K. Practical Guide to Vegetable Oil Processing (Second Edition), 2017, chapter 4, Pages 41-78.

${ }^{27}$ Mannu, A., Vlahopoulou, G., Sireus, V., Petretto, G.L., Mulas, G., Garroni, S. 2018. Bentonite as a Refining Agent in Waste Cooking Oils Recycling: Flash Point, Density and Color Evaluation. Nat. Prod. Commun., 13(5), 613-616.

28 Mannu, A., Vlahopoulou, G., Sireus, V., Mulas, G., Petretto, G.L. 2019. Characterization of Sardinian Bentonite. J. Sci. Res., 11(1), 145-150. https://doi.org/10.3329/jsr.v11i1.36900.

29 Czechlowski, M., Marcinkowski, D., Golimowska, R., Berger, W.A., Golimowski, W. 2019. Spectroscopy approach to methanol detection in waste fat methyl esters. Spectrochimica Acta Part A: Molecular and Biomolecular Spectroscopy, 210, 14-20. https://doi.org/10.1016/j.saa.2018.11.003.

30 Vlahopoulou, G., Petretto, G.L., Garroni, S., Piga, C., Mannu, A. 2018. Variation of density and flash point in acid degummed waste cooking oil. J. Food Process and Preservation, e13533. https://doi.org/10.1111/jfpp.13533.

31 Choe, E., Min, D.B. 2007. Chemistry of Deep-Fat Frying Oils. Journal of Food Science, 72, R77-R86. https://doi.org/10.1111/j.1750-3841.2007.00352.x.

32 Pokorny, J. Flavor chemistry of deep fat frying in oil. In: Min, D.B., Smouse, T.H., Zhang, S.S. (Eds.), Flavor Chemistry of Lipid Foods. American Oil Chemists Society, Champaign, 1989, 113-154. 
${ }^{33}$ Dogan, T.H. 2016. The testing of the effects of cooking conditions on the quality of biodiesel produced from waste cooking oils, Renewable Energy, 94, 466-473. https://doi.org/10.1016/j.renene.2016.03.088.

34 Van Del Dool H., Kartz, P.D. 1963. A generalization of the retention index system including linear temperature programmed gas-liquid partition chromatography. Journal of Chromatography A, 11, 463-471. https://doi.org/10.1016/S0021-9673(01)80947-X.

${ }^{35}$ Chong, J., Soufan, O., Li, C., Caraus, I., Li, S., Bourque, G., Wishart, D.S., Xia, J. 2018. MetaboAnalyst 4.0: towards more transparent and integrative metabolomics analysis. Nucl. Acids Res., 46, W486-494. https://doi.org/10.1093/nar/gky310.

${ }^{36}$ See Supporting Information for details.

37 See Supporting Information.

${ }^{38}$ Aparicio-Ruiz R., Romero I., García-González D.L., Oliver-Pozo C., Aparicio R. 2017. Soft-deodorization of virgin olive oil: Study of the changes of quality and chemical composition. Food Chemistry, 220, 42-50. https://doi.org/10.1016/j.foodchem.2016.09.176.

${ }^{39}$ Laoretani D.S., Iribarren O.A. 2017. Enhancing the productivity of batch deodorizers for edible oils. Journal of Food Engineering, 192, 72-78. https://doi.org/10.1016/j.jfoodeng.2016.08.004.

${ }^{40}$ Choe E., Min D.B. 2007. Chemistry of Deep-Fat Frying Oils. Journal of Food Science, 72, R77-R86. https://doi.org/10.1111/j.1750-3841.2007.00352.x.

${ }^{41}$ Zhang Q., Liu C., Sun Z., Hu X., Shen Q., Wu J. 2012. Authentication of edible vegetable oils adulterated with used frying oil by Fourier Transform Infrared Spectroscopy. Food Chemistry, 132(3), 1607-1613. https://doi.org/10.1016/j.foodchem.2011.11.129.

42 Alencar J.W., Alves P.B., Craveiro A.A., 1983. Pyrolysis of tropical vegetable oils. Journal of Agriculture and Food Chemistry, 31, 1268-1270. https://doi.org/10.1021/jf00120a031

43 Wu C. M., Chen S.Y. 1992. Volatile compounds in oils after deep frying or stir frying and subsequent storage. J. Am. Oil. Chem. Soc., 69, 858-65.

44 Dais, P., Spyros, A., Christophoridou, S., Hatzakis, E., Fragaki, G., Agiomyrgianaki, A., Salivaras, E., Siragakis, G., Daskalaki, D., Tasioula-Margari, M., Brenes, M. 2007. Comparison of Analytical Methodologies Based on ${ }^{1} \mathrm{H}$ and ${ }^{31} \mathrm{P}$ NMR Spectroscopy with Conventional Methods of Analysis for the Determination of Some Olive Oil Constituents. J. Agric. Food Chem., 55(3), 577-584. https://doi.org/10.1021/jf061601y.

45 Siciliano, C., Belsito, E., De Marco, R., Di Gioia, M.L., Leggio, A., Liguori, A. 2013. Quantitative determination of fatty acid chain composition in pork meat products by high resolution ${ }^{1} \mathrm{H}$ NMR spectroscopy. Food Chem., 136(2), 546-554. https://doi.org/10.1016/j.foodchem.2012.08.058.

46 Jiang, X., Wu, R.H.S., Wang, Q., Zhang, Z. 2018. Correlations between ${ }^{1} \mathrm{H}$ NMR and conventional methods for evaluating soybean oil deterioration during deep frying. Journal of Food Measurement and Characterization, 12, 1420-1426.

47 Hatzakis, E., Agiomyrgianaki, A., Kostidis, S. 2011. Dais, P. High-Resolution NMR Spectroscopy: An Alternative Fast Tool for Qualitative and Quantitative Analysis of Diacylglycerol (DAG) Oil. J. Am. Oil. Chem. Soc., 88(11), 1695-1708. https://doi.org/10.1007/s11746-011-1848-2.

${ }^{48}$ Vigli, G., Philippidis, A., Spyros, A., Dais, P. 2003. Classification of Edible Oils by Employing ${ }^{31} \mathrm{P}$ and ${ }^{1} \mathrm{H}$ NMR Spectroscopy in Combination with Multivariate Statistical Analysis. A Proposal for the Detection of Seed Oil Adulteration in Virgin Olive Oils. J. Agric. Food Chem., 51, 5715-5722. https://doi.org/10.1021/jf030100z 
49 Camin, F., Pavone, A., Bontempo, L., Wehrens, R., Paolini, M., Faberi, A., Marianella, R.M., Capitani, D., Vista, S., Mannina, L. 2016. The use of IRMS, ${ }^{1} \mathrm{H}$ NMR and chemical analysis to characterise Italian and imported Tunisian olive oils. Food Chem., 196, 98-105. https://doi.org/10.1016/j.foodchem.2015.08.132

50 Castejón, D., Mateos-Aparicio, I., Molero, M.D., Cambero, M.I., Herrera, A. 2014. Evaluation and Optimization of the Analysis of Fatty Acid Types in Edible Oils by ${ }^{1} \mathrm{H}-\mathrm{NMR}$. Food Anal. Methods, 6, $1285-1297$. https://doi.org/10.1007/s12161-013-9747-9.

51 Corsaro, C., Mallamace, D., Vasi, S., Ferrantelli, V., Dugo, G., Cicero, N. 2015. 'H HR-MAS NMR Spectroscopy and the metabolite determination of typical foods in mediterranean diet. J. Anal. Methods Chem., 1-14. https://doi.org/10.1155/2015/175696.

52 Liu, W., Yang, H., Wang, Z., Liu, J. 2012. Tracing the Source of Cooking Oils with an Integrated Approach of Using Stable Carbon Isotope and Fatty Acid Abundance. J. Agric. Food Chem., 60(32), 8069-8073. https://doi.org/10.1021/jf302410b.

53 Popescu, R., Costinel, D., Dinca, O.R., Marinescu, A., Stefanescu, I., Ionete, R.E. 2015. Discrimination of vegetable oils using NMR spectroscopy and chemometrics. Food Control., 48, 84-90. https://doi.org/10.1016/j.foodcont.2014.04.046.

${ }^{54} \Delta \mathrm{P}$ represents the difference between the upstream and downstream pressure, and $\mathrm{R}$ is the resistance of the medium.

${ }^{55}$ According to EN 314 Class 3, UNI 6478/69 M100, and DIN AW100. 